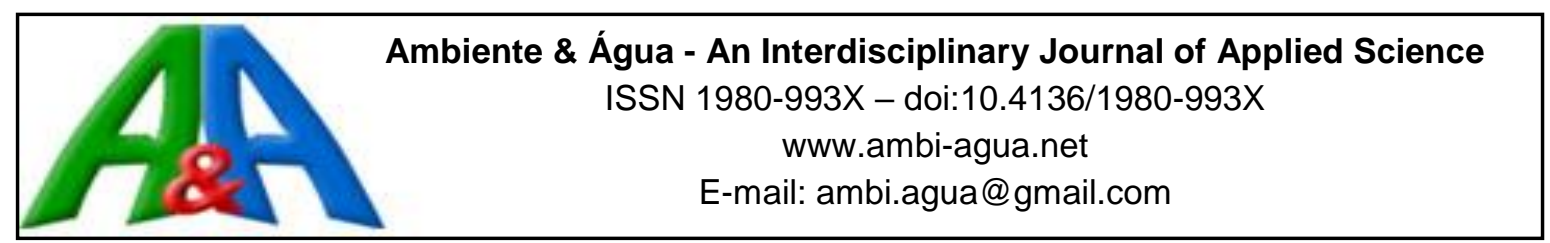

\title{
Incertezas e erros na estimativa de vazões usando modelagem hidrológica e precipitação por RADAR
}

\author{
doi:10.4136/ambi-agua.1924
}

Received: 20 Apr. 2016; Accepted: 19 Oct. 2016

\author{
Samuellson Lopes Cabral ${ }^{*}$; Jojhy Sakuragi ${ }^{1}$; Cleiton da Silva Silveira ${ }^{2}$ \\ ${ }^{1}$ Centro Nacional de Monitoramento e Alertas de Desastres Naturais (CEMADEN), \\ São José dos Campos, SP, Brasil \\ Coordenação de Operações e Modelagens \\ ${ }^{2}$ Universidade da Integração Internacional da Lusofonia Afro-Brasileira (UNILAB), \\ Redenção, Ceará, Brasil \\ *Autor correspondente: e-mail: samuellson.cabral@cemaden.gov.br, \\ jojhy.sakuragi@cemaden.gov.br, cleitonsilveira@unilab.edu.br
}

\section{RESUMO}

Esse artigo apresenta uma análise das incertezas e erros do modelo SCS-CN do Hydrologic Engineering Center - Hydrologic Modeling System (HEC-HMS), para eventos hidrológicos com dados de precipitação observada e estimada por RADAR na bacia do rio São Miguel em Alagoas, que controla uma área de $296 \mathrm{~km}^{2}$. Foram utilizados dados fisiográficos para as estimativas iniciais dos parâmetros do SCS-CN. Em seguida, utilizando medidas simultâneas de precipitação e vazões observadas, os parâmetros foram calibrados. Utilizando-se dados independentes, os parâmetros calibrados foram validados. O coeficiente de desempenho mostrou valores de Nash-Sutcliffe de 0,93 na fase de calibração e valores entre 0,81 a 0,87 nas fases de validação. Em seguida, o modelo foi utilizado para eventos hidrológicos passados, usando dados de precipitação estimada por RADAR. Obteve-se coeficientes de Nash-Sutcliffe entre 0,75 a 0,79 . O SCS-CN com as estimativas de precipitação pelo RADAR representou de forma adequada o tempo das vazões de pico, entretanto, subestimou a magnitude do pico com erros de até $26 \%$ em alguns eventos. A metodologia mostrou-se satisfatória para a bacia em estudo e pode ser uma ferramenta útil para aplicação em outras bacias hidrográfica prevendo possíveis inundações.

Palavras-chave: gestão dos recursos hídricos, inundação, SCS.

\section{Uncertainties and errors flow estimate using hydrological modelling and precipitation by RADAR}

\begin{abstract}
This work presents an analysis of uncertainties and errors of the SCS-CN model of the Hydrologic Engineering Center - Hydrologic Modeling System (HEC-HMS), for hydrological events using observed and RADAR-estimated precipitation data of the São Miguel River Basin in State of Alagoas, which covers an area of $296 \mathrm{~km}^{2}$. Physiographic data of the basin were used to find the initial estimates of the SCS-CN parameters. Then, using simultaneous measurements of precipitation and observed flow, the parameters were calibrated. Using
\end{abstract}


independent data series, calibrated parameters were validated. The performance coefficient showed Nash-Sutcliffe values of 0.93 in the calibration phase and between 0.81 and 0.87 in the validation phase. The model was then used for past hydrological events, using RADARestimated precipitation data. This resulted in Nash-Sutcliffe coefficients between 0.75 and 0.79. The SCS-CN model with RADAR-estimated precipitation represented the time of the peak flow adequately; however, it underestimated the magnitude of the peak with errors up to $26 \%$ in some events. The methodology had satisfactory results for this basin and can be a useful tool for the prediction of flooding in other watersheds.

Keywords: flooding, SCS, water resources management.

\section{INTRODUÇÃO}

A modelagem hidrológica é uma importante ferramenta no planejamento e gerenciamento de programas de recursos hídricos de bacias hidrográficas, auxiliando a previsão hidrológica. Os modelos hidrológicos necessitam de dados fisiográficos e dados temporais de chuva e vazão para uma melhor representação dos processos.

A análise de incertezas em modelos hidrológicos tem sido objeto de estudo de vários pesquisadores que assinalam a importância de se conduzir esse procedimento para que se tenham modelos que, de fato, representem mais acuradamente a realidade (Beck, 1987; McIntyre e Wheater, 2004; Muleta e Nicklow, 2005; Oreskes et al.,1994).

A incerteza na modelagem surge devido às informações incompletas usadas na simulação que estão na estrutura do programa de modelagem, nos parâmetros e nos dados de entrada. Em face disso, os autores sugerem que cada esforço de modelagem seja acompanhado de uma análise de incertezas com o objetivo de se determinar os intervalos de confiança das predições do modelo, levando em conta as várias fontes de incerteza. Esse procedimento é altamente recomendável quando a modelagem é aplicada, especialmente, para a tomada de decisão em políticas públicas.

Embora existam várias fontes de incertezas que afetam a precisão da resposta hidrológica em bacias hidrográficas, a precipitação é sem duvida umas das principais fontes de incertezas (Krzysztofowicz, 1999; 2001; Hossain e Anagnostou, 2004; Hossain et al., 2004; Kavetski et al., 2006a; 2006b; Nikolopoulos et al., 2010).

Segundo Cabral et al. (2016), quantificar a distribuição das precipitações sobre a terra é de suma importância para a compreensão da hidrologia de superfície. Essa quantificação pode desempenhar um importante papel na previsão e no monitoramento de fenômenos hidrometeorológicos. Entretanto, muitas das áreas propensas a esses fenômenos sofrem com a falta de dados pluviométricos confiáveis e sem falhas em sua série.

Radares meteorológicos permitem uma boa amostragem espacial e temporal da estimativa da taxa de precipitação, mas, por causa das fontes de erro, apresentam uma incerteza maior do que uma rede de pluviômetros.

Assim, tanto as medições de chuva com a rede de pluviômetros quanto às estimativas de chuva com o radar meteorológico possuem erros. As fontes de erro de medição de chuva com o radar meteorológico estão associadas à calibração eletrônica do equipamento, à equação de transformação da refletividade efetiva em taxa de precipitação (ou relação Z-R), ao efeito da curvatura da Terra, à zona de derretimento de cristais de gelo na nuvem, entre outras (Gonçalves, 2005). Da mesma forma, as medidas de chuva por pluviômetros não possuem representatividade espacial e a ação do vento pode causar uma diminuição da coleta de gotas pelo pluviômetro, entre outras.

Apesar dos erros de medição do radar e dos pluviômetros não serem bem conhecidos, as estimativas de chuva com o radar meteorológico e as medições de chuva com a rede de 
superfície podem ser combinadas para diminuir esses erros.

Sendo assim este artigo tem o objetivo de analisar as incertezas e erros na estimativa das vazões na bacia hidrográfica do rio São Miguel em Alagoas, utilizando dados de precipitações observadas e estimadas por RADAR como entrada em um modelo hidrológico SCS-CN do HEC-HMS.

\section{MATERIAL E MÉTODOS}

A bacia de drenagem do rio São Miguel está situada entre a Zona da Mata e o Agreste Alagoano (Figura 1). A bacia drena uma área de $624 \mathrm{~km}^{2}$, ao longo do curso de $90 \mathrm{~km}$ de extensão que perfaz o seu canal principal.

A estação chuvosa está entre o outono-inverno, com chuvas de março que ocorrem vindas do norte, e chuvas do principal período de sudeste, de abril-maio até junho-julho, mas com algumas interrupções. A montante da bacia, a precipitação anual varia de 1.100 a 1.400 $\mathrm{mm}$, e na região de foz ultrapassa o valor máximo anterior: $1.400 \mathrm{~mm}^{\mathrm{ano}}{ }^{-1}$.

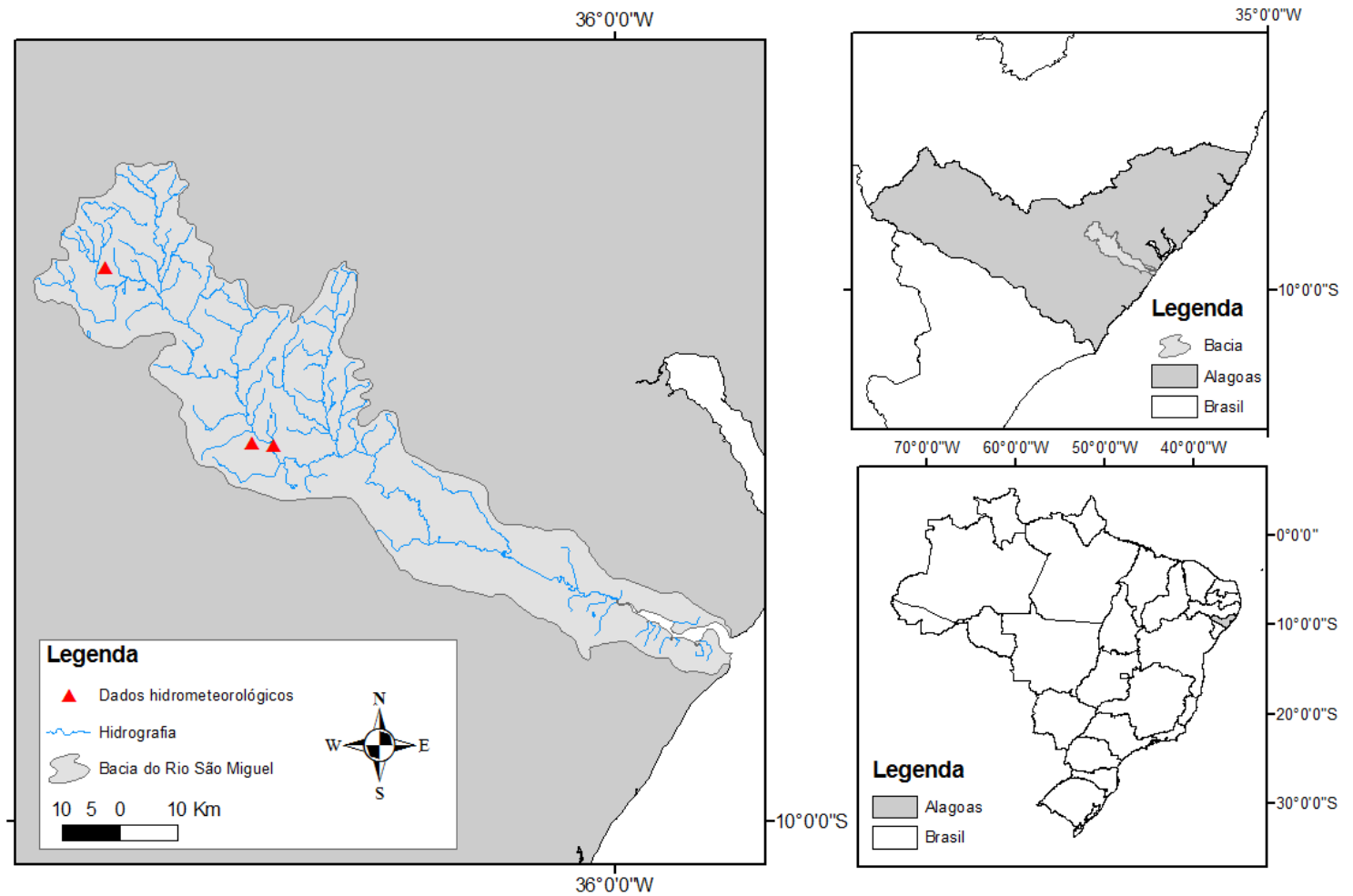

Figura 1. Localização da área de estudo.

\subsection{Dados pluviométricos e fluviométricos}

Para esse estudo foram utilizados dados de precipitação em escala diária, disponibilizados pela Secretaria de Estado de Meio Ambiente e Recursos Hídricos de Alagoas em www.semarh.al.gov.br e pela Agência Nacional de Águas em www.hidroweb.ana.gov.br.

Para o cálculo da precipitação média na bacia foi utilizado o método do polígono de Thiessen, conforme a Equação 1.

$$
\bar{P}=\frac{\sum P_{i} \cdot A_{i}}{A}
$$


em que:

$\mathrm{P}_{\mathrm{i}}$ : precipitações nos postos i $(\mathrm{mm})$;

$\mathrm{A}_{\mathrm{i}}$ : áreas de influência dos postos; e

A: área total da bacia.

Foram utilizados dados de uma estação fluviométrica da Agência Nacional de Águas (ANA) disponível em www.hidroweb.ana.gov.br, inserida na bacia hidrográfica do rio São Miguel para calibração e validação do modelo hidrológico.

A Tabela 1 apresenta os eventos hidrológicos selecionados para esse estudo, com destaque nas datas iniciais e finais dos eventos, assim como o tempo de duração e as vazões máximas dos eventos.

Tabela 1. Eventos hidrológicos selecionados.

\begin{tabular}{ccccc}
\hline Evento & $\begin{array}{c}\text { Data } \\
\text { Início }\end{array}$ & $\begin{array}{c}\text { Data } \\
\text { Fim }\end{array}$ & $\begin{array}{c}\text { Tempo } \\
\text { (horas) }\end{array}$ & $\begin{array}{c}\text { Vazão máxima } \\
\left(\mathrm{m}^{3} \mathrm{~s}^{-1}\right)\end{array}$ \\
\hline 1 & $02 / 05 / 14$ & $02 / 05 / 14$ & 6,0 & 43,07 \\
2 & $22 / 05 / 14$ & $23 / 05 / 14$ & 12,5 & 29,27 \\
3 & $07 / 08 / 14$ & $08 / 08 / 14$ & 16,0 & 70,06 \\
4 & $05 / 10 / 14$ & $07 / 10 / 14$ & 12,5 & 53,08 \\
5 & $28 / 06 / 15$ & $29 / 06 / 15$ & 12,0 & 37,72 \\
\hline
\end{tabular}

\subsection{Dados do RADAR}

Foram utilizados os dados do radar meteorológico do Centro Nacional de Monitoramento e Alertas de Desastres Naturais (CEMADEN) de Maceió/AL, localizado em $35.77^{\circ} \mathrm{W}$ e $9.55^{\circ} \mathrm{S}$, banda $\mathrm{S}$, doppler e de dupla polarização. Os pontos de grade do RADAR tem resolução espacial de $250 \mathrm{~m}$, que cobre a área da bacia com oito pontos.

A metodologia utilizada na obtenção da precipitação acumulada consiste em aplicar as variáveis polarimétricas $(\mathrm{Z}$ - refletividade horizontal [dBZ], $\mathrm{Zdr}$ - refletividade diferencial [dB] e Kdp - diferencial do deslocamento de fase $[\% / \mathrm{km}]$ ) conforme os limiares de taxa de precipitação (Equações 2, 3, 4, 5, e 6) para a obtenção da precipitação instantânea segundo a proposta por Ryzhkov et al. (2005). A cada varredura (10 minutos) é feita essa estimativa e os resultados são acumulados em períodos de 24-h. $\mathrm{R}(Z)$ é a relação de Marshall e Palmer (1948), onde $Z=a R b$, em que a e b são constantes iguais a 200 e 1.6, respectivamente.

$$
\mathrm{R}=\frac{\mathrm{R}(\mathrm{Z})}{0.4+5.0\left|\mathrm{Z}_{\mathrm{dr}}-1\right|^{1.3}}
$$

para $\mathrm{R}(\mathrm{Z})<6 \mathrm{~mm} \mathrm{~h}^{-1}$

$$
R=\frac{R\left(K_{d p}\right)}{0.4+3.5\left|Z_{d r}-1\right|^{1.7}}
$$

para $6<\mathrm{R}(\mathrm{Z})<50 \mathrm{~mm} \mathrm{~h}^{-1}$ 


$$
\mathrm{R}=\mathrm{R}\left(\mathrm{K}_{\mathrm{dp}}\right)
$$

para $\mathrm{R}(\mathrm{Z})>50 \mathrm{~mm} \mathrm{~h}^{-1}$

em que:

$$
\begin{aligned}
& \mathrm{R}(\mathrm{Z})=\frac{1}{a^{1 / b}} Z^{1 / b} \Leftrightarrow Z=a R^{b} \\
& \mathrm{R}\left(\mathrm{K}_{\mathrm{dp}}\right)=44.0\left|K_{d p}\right|^{0.822}
\end{aligned}
$$

para KDP $>0$

Uma calibração prévia da precipitação estimada por radar foi realizada utilizando dados das estações pluviométricas dentro da área de varredura quantitativa, seguindo os métodos de Brandes (1975) e Barnes (1964), onde foi detectada a priori uma subestimativa de 60\%, na média. Assim, esta correção foi aplicada nesse trabalho para alimentar o modelo hidrológico.

\subsection{Modelagem hidrológica: SCS-CN do HEC-HMS}

O HEC-HMS é um programa que pode ser adaptado a um sistema de interesse, através de mudanças nos dados em um banco de dados ou alterações nos parâmetros, nas condições de contorno e nas condições de entrada iniciais (Campos, 2009).

Para o cálculo do hietograma, utilizou-se o método dos blocos alternados. Na determinação da precipitação efetiva, aplicou-se o método da Curve Number $(\mathrm{CN})$, desenvolvido pelo Natural Resources Conservation Service (NRCS).

$\mathrm{O}$ valor de $\mathrm{CN}$ responde pela maior parte das características das bacias hidrográficas produtoras de escoamento, tais como tipo de solo, uso da terra, condição hidrológica e condição antecedente de umidade. (Mishra e Singh, 2004).

Para estimar a precipitação efetiva, aplicou-se a Equação 7:

$$
\mathrm{P}_{\mathrm{e}}=\frac{\left(\mathrm{P}-\mathrm{I}_{\mathrm{a}}\right)^{2}}{\mathrm{P}-\mathrm{I}_{\mathrm{a}}+\mathrm{S}} \text { para } \mathrm{P}>\text { Ia e } 0 \text { nos demais casos }
$$

em que:

$$
\begin{aligned}
& \mathrm{P}_{\mathrm{e}} \text { : é chuva efetiva }(\mathrm{mm}) ; \\
& \mathrm{P} \text { : é precipitação total }(\mathrm{mm}) ; \\
& \mathrm{I}_{\mathrm{a}} \text { : é abstração inicial }(\mathrm{mm}) ; \text { e } \\
& \mathrm{S} \text { : é máximo potencial de retenção do solo }(\mathrm{mm}) .
\end{aligned}
$$

Para Ia adotou-se o valor de 0,2S, conforme recomendado pelo NRCS. Assim, a Equação 7 pode ser reescrita na forma da Equação 8.

$$
\mathrm{P}_{\mathrm{e}}=\frac{(\mathrm{P}-0.2 \mathrm{~S})^{2}}{\mathrm{P}+0.8 \mathrm{~S}}
$$

em que:

S obtido pela Equação 9. 


$$
\mathrm{S}=\frac{(1000)}{\mathrm{CN}}-10
$$

$\mathrm{O}$ parâmetro $\mathrm{CN}$ está relacionado ao tipo de solo, ao tipo de cobertura vegetal e às condições de umidade antecedentes. Nesse estudo utilizou-se condição antecedente de umidade média para um solo tipo B com cobertura vegetal tipo: urbano, área vegetada e mata ciliar. Na estimativa do $\mathrm{CN}$, foram utilizadas imagens aéreas do município, combinadas com imagens do Google Earth. Destaca-se, que o valor do número $\mathrm{CN}$ foi estimado a partir dos valores da tabela determinada pelo NRCS, conforme o tipo de solo da área de estudo.

Para obter o hidrograma da cheia a partir do hietograma da chuva, aplicou-se o método do Hidrograma Unitário Adimensional do NRCS, seguindo a proposta de Ebrahimian et al. (2012). O método tem como parâmetro o tempo de retardo $\left(\mathrm{T}_{\mathrm{lag}}\right)$. $\mathrm{O}$ valor de $\mathrm{T}_{\mathrm{lag}}$ é obtido pela Equação 10.

$$
\mathrm{T}_{\text {lag }}=0.6 \mathrm{~T}_{\mathrm{c}}
$$

em que:

Tc: é tempo de concentração (min).

Para cálculo, o Tc é estimado pela Equação de Kirpich, Equação 11.

$$
\mathrm{T}_{\mathrm{c}}=0.0078 \mathrm{x}\left(\frac{\mathrm{L}^{0.77}}{\mathrm{~S}^{0.385}}\right)
$$

em que:

L: comprimento do rio $(\mathrm{km})$; e

S: declividade do rio $(\mathrm{m} / \mathrm{m})$.

A Tabela 2 apresenta algumas características fisiográficas (área da bacia, comprimento do rio, lag time, tempo de concentração, altitude e declividade média) obtidas através de técnicas de Sistema de Informações Geográficas (SIG) e programas computacionais.

Tabela 2. Característica fisiográfica da sub-bacia do rio São Miguel.

\begin{tabular}{ccccccc}
\hline Bacia & $\begin{array}{c}\text { Área } \\
\left(\mathrm{km}^{2}\right)\end{array}$ & $\begin{array}{c}\text { Comprimento } \\
\text { do rio }(\mathrm{km})\end{array}$ & $\begin{array}{c}\text { Lag Time } \\
(\mathrm{h})\end{array}$ & $\begin{array}{c}\text { Tempo de } \\
\text { Concentração }(\mathrm{h})\end{array}$ & $\begin{array}{c}\text { Altitude Média } \\
(\mathrm{m})\end{array}$ & $\begin{array}{c}\text { Declividade } \\
\text { Média }(\mathrm{m} / \mathrm{m})\end{array}$ \\
\hline São Miguel & 296 & 41 & 2,07 & 3,45 & 480 & 0,00978 \\
\hline
\end{tabular}

\subsection{Parametrização multi objetivo}

Foram escolhidos eventos registrados pelo posto fluviométrico para calibração e validação das vazões com as chuvas observadas como entrada. O procedimento de calibração automática do HEC-HMS utiliza um método iterativo para minimizar/maximizar uma função objetivo.

Para a calibração do SCS-CN foi escolhida uma função objetivo baseada no erro percentual do pico (Equação 12), essa medida só considera a magnitude de computação total do pico do evento. 


$$
Z=\sum_{i=1}^{N Q}\left|\frac{q_{0}(\text { pico })-q_{s}(\text { pico })}{q_{0}(\text { pico })}\right|
$$

em que:

Z: é função objetiva;

NQ: são números de ordenadas do hidrogramas calculadas;

Qo $(\mathrm{t})$ : é vazão observada;

$\mathrm{Q}_{\mathrm{s}}(\mathrm{t})$ : são vazões calculadas com os parâmetros do modelo;

qo (pico): é pico observado;

qo (média): é média das vazões; e

$\mathrm{q}_{\mathrm{s}}$ (pico): é pico calculado.

\subsection{Análise da eficiência da modelagem hidrológica}

Para determinação da eficiência da modelagem foi utilizado o coeficiente de Nash-Sutcliffe (1970) que tem sido largamente utilizado na verificação do desempenho de modelos hidrológicos. O coeficiente NS pode ser dado pela seguinte Equação 13.

$$
N S=1-\frac{\sum_{t=1}^{n t}\left[Q_{o b s}(t)-Q_{\text {sim }}(t)\right]^{2}}{\sum_{t=1}^{n t}\left[Q_{o b s}(t)-\bar{Q}_{o b s}(t)\right]^{2}}
$$

em que:

$\mathrm{Q}_{\text {obs }}(\mathrm{t})$ : vazão observada no passo de tempo $\mathrm{t}$ em $\left(\mathrm{m}^{3} \mathrm{~s}^{-1}\right)$;

$\mathrm{Q}_{\text {sim }}(\mathrm{t})$ : vazão simulada em $\left(\mathrm{m}^{3} \mathrm{~s}^{-1}\right)$; e

Q obs: média de vazões observadas de todo o período de simulação em $\left(\mathrm{m}^{3} \mathrm{~s}^{-1}\right)$.

\section{RESULTADOS E DISCUSSÃO}

\subsection{Análises das vazões}

A Tabela 3 mostra o desempenho da estimativa das vazões usando o SCS-CN na fase de calibração e validação, com as precipitações observadas e estimadas com RADAR para os eventos selecionados, conforme a descrição dos eventos hidrológicos na Tabela 1. Nessa fase a modelagem hidrológica apresentou valores $\mathrm{NS}=0,93$ para o período da calibração.

Moriasi et al. (2007) destacam que os limites aceitáveis dos critérios de avaliação da calibração de modelos hidrológicos devem ser menos exigentes para simulações com passo de tempo diário. Segundo Collischonn (2001), valores de Nash-Sutcliffe > 0,75, como os encontrados nesse estudo, são considerados adequados.

Para os eventos hidrológicos 2, 3, 4 e 5, o modelo SCS-CN do HEC-HMS apresentou desempenho satisfatório, com valores de Nash-Sutcliffe entre 0,87 a 0,81 para vazões com a precipitação observada, e Nash-Sutcliffe entre 0,75 a 0,79 para vazões com estimativa de chuva do RADAR de Maceió-AL. 
Tabela 3. Eficiência da calibração dos parâmetros do SCS-CN.

\begin{tabular}{ccccc}
\hline Evento & $\begin{array}{c}\text { Períodos } \\
\text { (horas) }\end{array}$ & Fase & $\mathrm{Q}\left(\mathrm{P}_{\mathrm{obs}}\right)$ & $\mathrm{Q}\left(\mathrm{P}_{\text {RADAR }}\right)$ \\
\hline 1 & 6,0 & Calibração & 0,90 & - \\
2 & 12,5 & Validação & 0,87 & 0,79 \\
3 & 16,0 & Validação & 0,81 & 0,77 \\
4 & 12,5 & Validação & 0,83 & 0,75 \\
5 & 12,0 & Validação & 0,86 & 0,78 \\
\hline
\end{tabular}

A Figura 2 apresenta o hidrograma das vazões observadas e simuladas pelo SCS-CN do HEC-HMS, e o hietograma da precipitação média observada na bacia hidrográfica para o evento hidrológico utilizado na fase de calibração, verificando assim a eficiência da calibração do modelo. O SCS-CN mostrou boa consistência na parametrização e uma representação eficiente dos processos de propagação pelo modelo. Nota-se que o hidrograma ajustou-se bem tanto à vazão de pico quanto no período de recessão para todo o evento. Isso pode estar associado principalmente a função objetiva escolhida na parametrização dos parâmetros do modelo hidrológico. Nota-se que o modelo subestima as vazões no início do evento até vazões da ordem de $15 \mathrm{~m}^{3} \mathrm{~s}^{-1}$; já com valores maiores que $15 \mathrm{~m}^{3} \mathrm{~s}^{-1}$ o modelo tende a subestimar essas vazões. Entretanto de maneira geral o modelo representou de forma satisfatória as vazões, com valor de NS $=0,90$.

Segundo Sene (2012), algumas incertezas e erros podem está associadas a problemas nos dados pluviométricos, tais como: medição, obstrução ou falta de manutenção, bem como a imprecisão na distribuição espacial da precipitação ao longo da bacia hidrográfica.

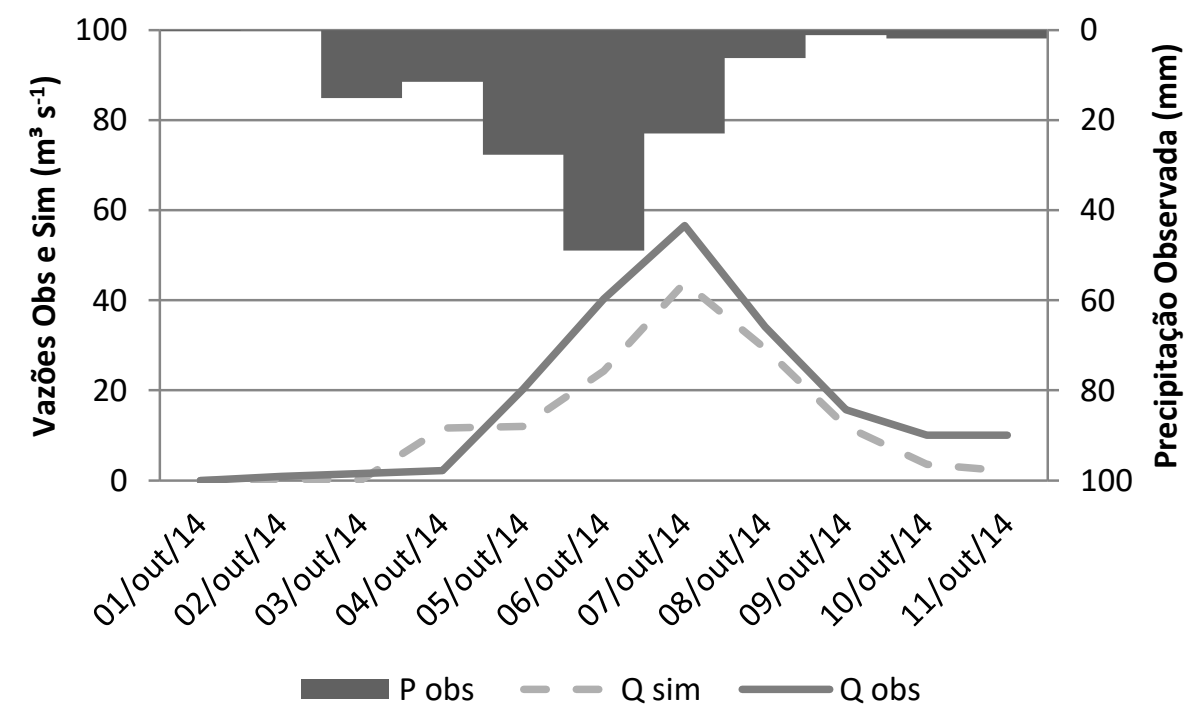

Figura 2. Hidrograma da vazão simulada e observada, e hietograma da precipitação observada.

A Figura 3 apresenta os hidrogramas das vazões observadas e simuladas pelo SCS-CN do HEC-HMS, com dados do RADAR na bacia hidrográfica do rio São Miguel, para os 
eventos hidrológicos (2(a), 3(b), 4(c) e 5(d)). O SCS-CN mostrou desempenho satisfatório para as vazões, com os dados de entrada de precipitação calibrados do RADAR de Maceió AL. O modelo conseguiu representar bem as curvas das vazões, entretanto não conseguiu representar as vazões do pico dos eventos, apresentando erros da ordem de até $26 \%$. Todavia, os resultados demonstram ser útil na representação de eventos de extremos hidrológicos na região.

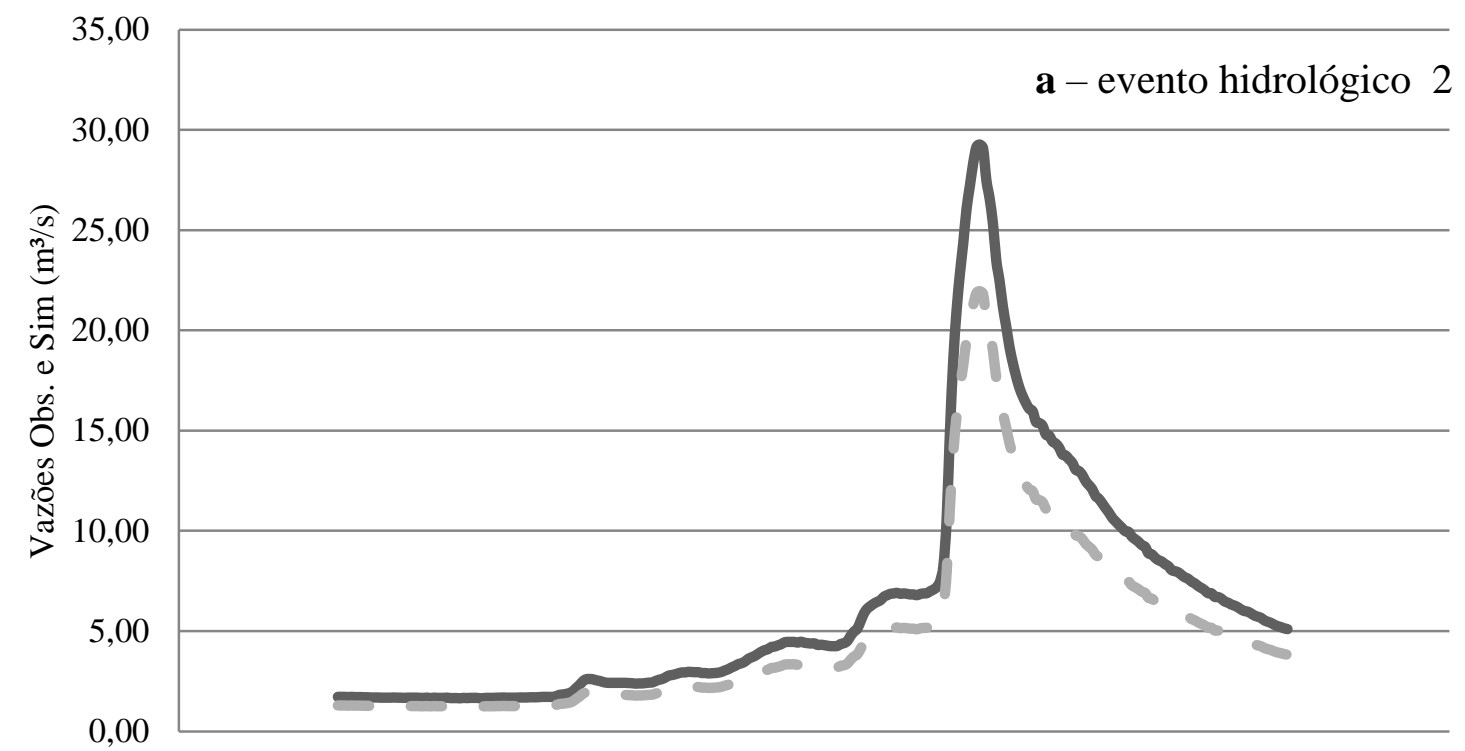

20/05/2014 21/05/2014 21/05/2014 22/05/2014 22/05/2014 23/05/2014 23/05/2014 24/05/2014 24/05/2014 Tempo (dias)

$\longrightarrow$ Vazão Obs $\left(\mathrm{m}^{3} / \mathrm{s}\right) \longrightarrow$ Vazão Sim RADAR $\left(\mathrm{m}^{3} / \mathrm{s}\right)$

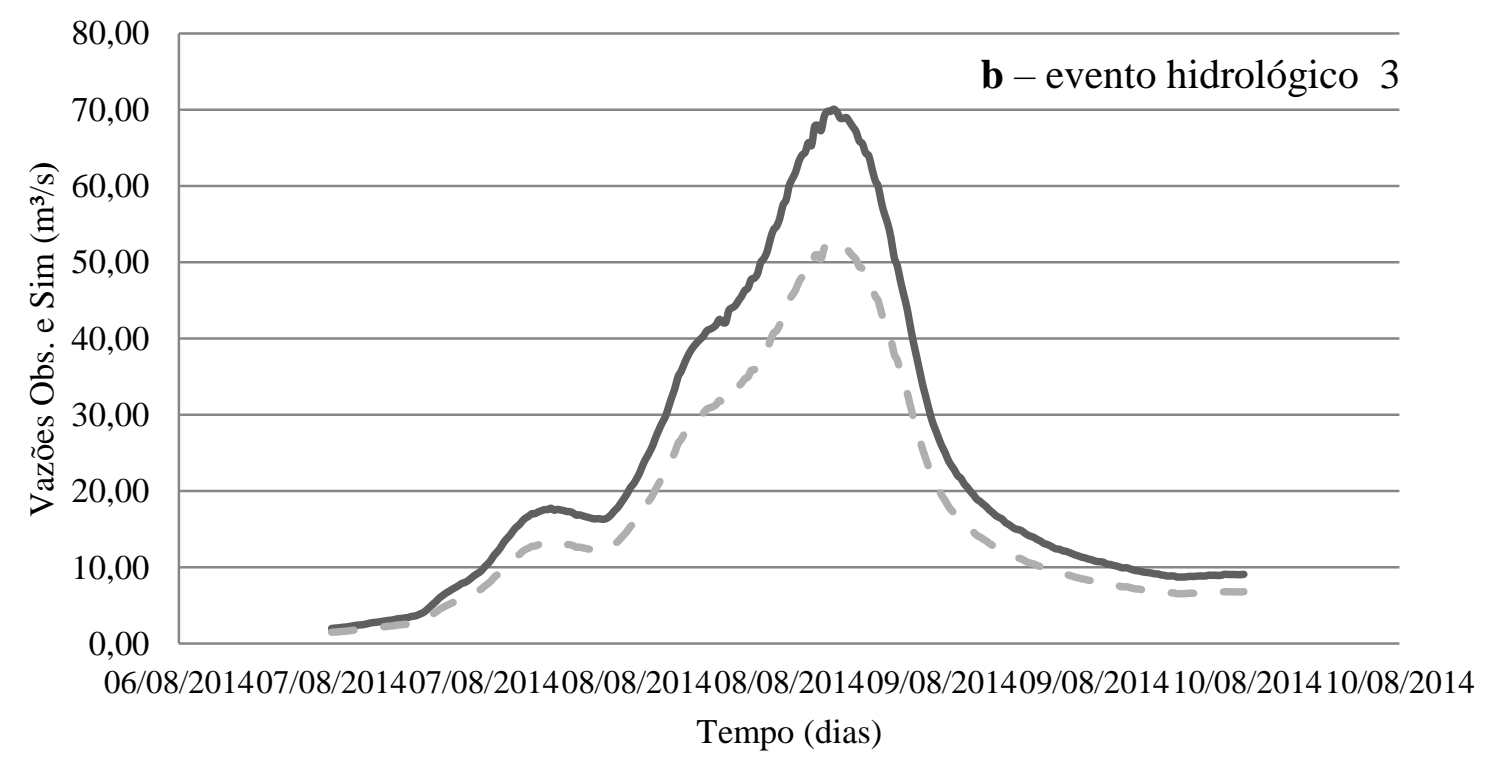

Vazão Obs $\left(\mathrm{m}^{3} / \mathrm{s}\right) \quad-$ Vazão $\operatorname{Sim} \operatorname{RADAR}\left(\mathrm{m}^{3} / \mathrm{s}\right)$ 


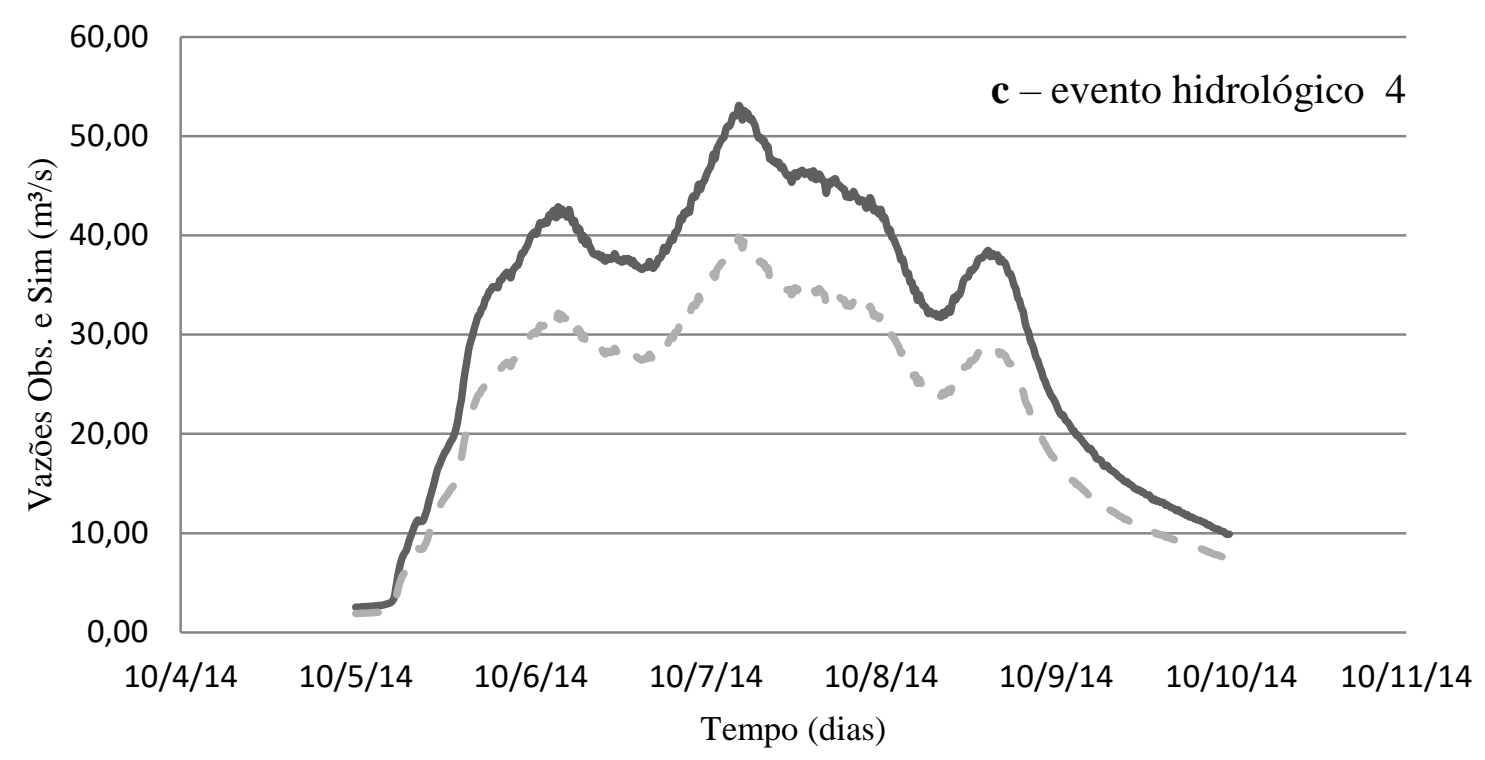

$\longrightarrow$ Vazão Obs $\left(\mathrm{m}^{3} / \mathrm{s}\right) \quad-$ Vazão $\operatorname{Sim} \operatorname{RADAR}\left(\mathrm{m}^{3} / \mathrm{s}\right)$

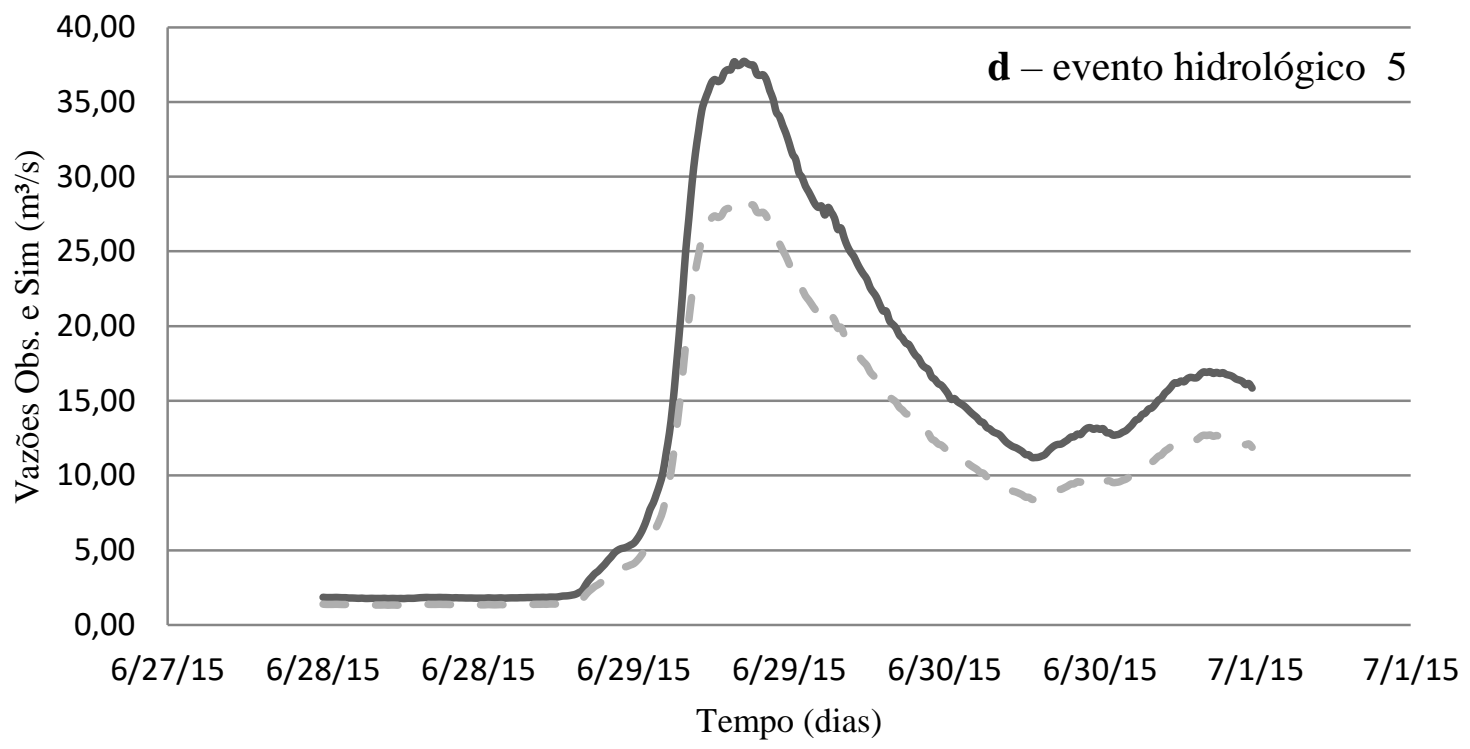

$\longrightarrow$ Vazão Obs $\left(\mathrm{m}^{3} / \mathrm{s}\right) \quad-$ Vazão $\operatorname{Sim} \operatorname{RADAR}\left(\mathrm{m}^{3} / \mathrm{s}\right)$

Figura 3. Hidrogramas das vazões simuladas e observadas nos eventos hidrológicos 2, 3, 4, e 5.

Verifica-se um desempenho superior, comparando com outros estudos que utilizaram o modelo HEC-HMS, (De Silva et al., 2014; Haberlandt e Radtke, 2014). Entretanto, em uma avaliação de três modelos hidrológicos na bacia hidrográfica do rio Una, no NEB, Carvalho Neto, Ribeiro Neto e Montenegro (2014) observaram desempenho semelhante entre o modelo HEC-HMS, um modelo concentrado (MODHAC) e um modelo distribuído por células (MGB-IPH).

A Tabela 4 mostra as análises do tempo do pico, das vazões de pico e o volume total com informações das simulações do SCS-CN para precipitação observada e pelo RADAR. Os tempos de pico das vazões máximas calculadas foram iguais aos observados para todos os casos. Embora o SCS-CN do HEC-HMS tenha conseguido acompanhar as vazões de pico dos 
eventos, tanto com as precipitações observadas como com as precipitações estimadas pelo RADAR, o modelo subestimou os valores de pico em todos os eventos simulados, com erros variando entre $3 \%$ a $26 \%$ para os eventos simulados. Os volumes estimados pelo modelo SCS-CN apresentaram erros para todos os eventos, com erros menores para a simulação usando precipitações observadas $(9,49 \%)$, e erros mais significativos para estimativas de precipitações por RADAR (40,87\%). Esses erros podem esta relacionados à distribuição espacial da precipitação observada e/ou à subestimação da precipitação por RADAR. Entretanto o método mostrou valores considerados satisfatórios, levando em consideração a limitação da base de dados observados e a qualidade dos dados na bacia hidrográfica.

Tabela 4. Análise das estimativas das vazões com precipitações observadas e do RADAR.

\begin{tabular}{|c|c|c|c|c|c|c|}
\hline \multirow{4}{*}{$\begin{array}{l}\overrightarrow{0} \\
\stackrel{0}{0} \\
\text { 齐 }\end{array}$} & & $\begin{array}{l}\text { Tempo do } \\
\text { pico }\end{array}$ & $\begin{array}{l}Q_{\text {pico }} \\
\left(\mathrm{m}^{3} \mathrm{~s}^{-1}\right)\end{array}$ & $\begin{array}{l}\text { Erro } Q_{\text {pico }} \\
\quad(\%)\end{array}$ & $\begin{array}{c}\text { Volume } \\
\text { (mm) }\end{array}$ & $\begin{array}{c}\text { Erro Volume } \\
\qquad(\%)\end{array}$ \\
\hline & $Q_{\text {obs }}$ & $02 / 05 / 14$ & 43,07 & - & 44,22 & - \\
\hline & $\operatorname{Sim}_{\text {(Pobs) }}$ & $02 / 05 / 14$ & 38,23 & 10,77 & 39,87 & 9,83 \\
\hline & $\begin{array}{c}\text { Sim } \\
\text { (RADAR) }\end{array}$ & $02 / 05 / 14$ & - & - & - & - \\
\hline \multirow{3}{*}{ 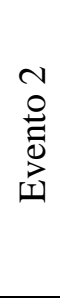 } & $Q_{\text {obs }}$ & $22 / 05 / 14$ & 29,27 & - & 31,18 & - \\
\hline & $\operatorname{Sim}_{(\mathrm{Pobs})}$ & $22 / 05 / 14$ & 26,55 & 9,29 & 28,22 & 9,49 \\
\hline & $\begin{array}{c}\text { Sim } \\
\text { (RADAR) }\end{array}$ & $22 / 05 / 14$ & 21,88 & 25,24 & 25,85 & 17,09 \\
\hline \multirow{3}{*}{$\begin{array}{l}m \\
\stackrel{0}{0} \\
\stackrel{0}{0} \\
\stackrel{2}{1} \\
0\end{array}$} & $Q_{\text {obs }}$ & 07/08/14 & 70,06 & - & 82,25 & - \\
\hline & $\operatorname{Sim}_{(\mathrm{Pobs})}$ & 07/08/14 & 67,33 & 3,89 & 75,39 & 8,34 \\
\hline & $\begin{array}{c}\text { Sim } \\
\text { (RADAR) }\end{array}$ & 07/08/14 & 52,54 & 25,0 & 64,37 & 21,73 \\
\hline \multirow{3}{*}{$\begin{array}{l}+ \\
\stackrel{0}{0} \\
\stackrel{0}{0} \\
\stackrel{D}{ \pm}\end{array}$} & $Q_{\text {obs }}$ & 05/10/14 & 53,08 & - & 54,43 & - \\
\hline & $\operatorname{Sim}_{(\mathrm{Pobs})}$ & $05 / 10 / 14$ & 43,90 & 17,29 & 40,26 & 26,03 \\
\hline & $\begin{array}{c}\mathrm{Sim} \\
\text { (RADAR) }\end{array}$ & 05/10/14 & 39,0 & 26,0 & 32,18 & 40,87 \\
\hline \multirow{3}{*}{$\begin{array}{l}n \\
\stackrel{0}{0} \\
\stackrel{0}{0} \\
\stackrel{1}{1}\end{array}$} & $Q_{\text {obs }}$ & $28 / 06 / 15$ & 37,72 & - & 41,16 & - \\
\hline & $\operatorname{Sim}_{\text {(Pobs) }}$ & $28 / 06 / 15$ & 34,85 & 7,60 & 39,12 & 6,09 \\
\hline & $\underset{(\operatorname{RADAR})}{\mathrm{Sim}}$ & $28 / 06 / 15$ & 28,29 & 25,0 & 35,82 & 14,01 \\
\hline
\end{tabular}

\section{IPABH}




\section{CONCLUSÃO}

A calibração e validação dos parâmetros do SCS-CN do HEC-HMS mostrou bom desempenho, validando o uso do modelo hidrológico na região de estudo. O SCS-CN mostrou um bom ajuste nas vazões simuladas para os eventos hidrológicos com as duas formas de precipitação (observada e RADAR), como dado de entrada no modelo.

O modelo hidrológico conseguiu demonstrar de forma satisfatória os eventos hidrológicos usando dados de RADAR, mesmo subestimando a magnitude das vazões de pico. O modelo representou de forma satisfatória a tendência das vazões, mostrando ser uma ferramenta que pode oferecer subsídios a fim de mitigar eventos extremos de caráter hidrológico na região.

Por fim, o presente trabalho poderá contribuir para melhorar o desempenho de modelos hidrológicos e auxiliar os sistemas de alerta a inundações, podendo ainda aplicar a correção da chuva para outras áreas de cobertura do radar, e assim, utilizar essa informação quantitativa em modelos hidrológicos, e também para diversas outras áreas onde se apresentam grandes carências por informações de precipitação com boa distribuição espacial e temporal.

\section{REFERENCIAS}

BARNES, S. L. A technique for maximizing details in numerical weather map analysis. Journal of Applied Meteorology, v. 3, p. 396-409, 1964. http://dx.doi.org/ 10.1175/1520-0450(1964)003\%3C0396:ATFMDI\%3E2.0.CO;2

BRANDES, E. A. Optimizing rainfall estimates with the aid of radar. Journal of Applied Meteorology, v. 14, p. 1339-1345, 1975. http://dx.doi.org/10.1175/15200450(1975)014<1339:OREWTA>2.0.CO;2

BECK, M. B. Water Quality modeling: a review of the analysis of uncertainty.Water Resources Research, v. 23, n. 8, p. 1393-1442, 1987. http://dx.doi.org/ 10.1029/WR023i008p01393

CABRAL, S. L.; CAMPOS, J. N. B.; SILVEIRA, C. S.; RODRIGUES, J. M. O intervalo de tempo para uma máxima previsibilidade da precipitação sobre o Semiárido brasileiro. Revista Brasileira de Meteorologia, v. 31, n. 2, 2016. http://dx.doi.org/10.1590/0102778631220130034

CAMPOS, J. N. B. Lições em modelos e simulação hidrológica. Fortaleza: ASTEF/Expressão Gráfica, 2009. v. 1. 166 p.

CARVALHO NETO, J. G.; RIBEIRO NETO, A.; MONTENEGRO, S. M. G. L. Assessment of RainfallRunoff Models for Flood River Extreme Event Simulations. In: INTERNATIONAL CONFERENCE ON FLOOD MANAGEMENT, 6., 2014, São Paulo. Anais... São Paulo; [s.n.], 2014.

COLLISCHONN, W. Simulação hidrológica em grandes bacias. 2001. 194f. Tese (Doutorado em Engenharia Civil) - Universidade Federal do Rio Grande do Sul, Porto Alegre, 2001.

DE SILVA, M. M. G. T.; WEERAKOON, S. B.; HERATH, S. Modeling of Event and Continuous Flow Hydrographs with HEC-HMS: Case Study in the Kelani River Basin, Sri Lanka. Journal of Hydrologic Engineering, v. 19, n. 4, p. 800-806, 2014. http://dx.doi.org/10.1061/(ASCE)HE.1943-5584.0000846 
GONÇALVES, F. M. Utilização de radar meteorológico em hidrologia. 2005. $116 f$. Dissertação (Mestrado em Engenharia) - Escola Politécnica da Universidade de São Paulo, São Paulo,2005.

HABERLANDT, U.; RADTKE, I. Hydrological model calibration for derived flood frequency analysis using stochastic rainfall and probability distributions of peak flows.

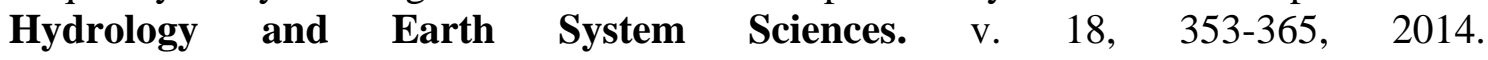
http://dx.doi.org/10.5194/hess-18-353-2014

HOSSAIN, F.; ANAGNOSTOU, E. N. Assessment of current passive-microwaveand infrared-based satellite rainfall remote sensing for flood prediction. Journal of Geophysical Research: Atmospheres, v. 109, n. D7, 2004. http://dx.doi.org/10.1029/2003JD003986

HOSSAIN, F.; ANAGNOSTOU, E. N.; DINKU, T.; BORGA, M. Hydrological model sensitivity to parameter and radar rainfall estimation uncertainty. Hydrological Processes, v. 18, n. 17, p. 3277-3291, 2004. http://dx.doi.org/10.1002/hyp.5659

KAVETSKI, D.; KUCZERA, G.; FRANKS, S. W. Bayesian analysis of input uncertainty in hydrological modeling: 1. theory. Water Resources Research, v. 42, n. 3, 2006. http://dx.doi.org/10.1029/2005WR004376

KAVETSKI, D.; KUCZERA, G.; FRANKS, S. W. Bayesian analysis of input uncertainty in hydrological modeling: 2. application. Water Resources Research, v. 42, n. 3, 2006. http://dx.doi.org/10.1029/2005WR004376

KRZYSZTOFOWICZ, R. Bayesian theory of probabilistic forecasting via deterministic hydrologic model. Water Resources Research, v. 35, n. 9, p. 2739-2750, 1999. 10.1029/1999WR900099

KRZYSZTOFOWICZ, R. The case for probabilistic forecasting in hydrology. Journal of Hydrology, v. 249, p. 2-9, 2001. http://dx.doi.org/10.1016/S0022-1694(01)00420-6

MARSHALL, J. S.; PALMER, W. Mc K. The distribution of raindrops with size. Journal of Meteorology, v. 5, p. 165-166, 1948. http://dx.doi.org/10.1175/15200469(1948)005\%3C0165:TDORWS\%3E2.0.CO;2

MCINTYRE, N.; WHEATER, H. S. Calibration of an in-river phosphorus model: prior evaluation of data needs and model uncertainty. Journal of Hydrology, v. 290, p. 100116, 2004. http://dx.doi.org/10.1016/j.jhydrol.2003.12.003

MISHRA, S. K.; SINGH, V. P. Long-term hydrological simulation based on the Soil Conservation Service curve number. Hydrological Processes, v. 18, p. 1291-1313. http://dx.doi.org/10.1002/hyp.1344

MORIASI, D. N.; ARNOLD, J. G.; VANLIEW, M. W.; BINGNER, R. L.; HARMEL, R. D. VEITH, T. L. Model evaluation guidelines for systematic quantification of accuracy in watershed simulations. Transactions of the ASABE, v. 50, n. 3, p. 885-900, 2007. $10.13031 / 2013.23153$

MULETA, M. K.; NICKLOW, J. W. Sensitivity and uncertainty analysis coupled with automatic calibration for a distributed watershed model. Journal of Hydrology, v. 306, p. 127-145, 2005. http://dx.doi.org/10.1016/j.jhydrol.2004.09.005 
NIKOLOPOULOS, E. I.; ANAGNOSTOU, E. N.; HOSSAIN, F.; GEBREMICHAEL, M.; BORGA, M. Understanding the scale relationships of uncertainty propagation of satellite rainfall through a distributed hydrologic model. Journal of Hydrometeorology, American Meteorological Society, v. 11, n. 2, p. 520-532, 2010. http://dx.doi.org/10.1175/2009JHM1169.1

ORESKES, N.; SHRADER-FRECHETTE, K.; BELITZ, K. Verification. validation and confirmation of numerical models in the earth sciences. Science, v. 263, p. 641-646, 1994.

RYZHKOV, A. V.; GIANGRANDE, S. E.; SCHUUR, T. J. Rainfall estimation with a polarimetric prototype of WSR-88D. Journal Applied Meteorology, v. 44, p. 502-515, 2005. http://dx.doi.org/10.1175/JAM2213.1

SENE, K. Flash Floods- Forecasting and Warning. Dordrecht: Springer, 2012.168p. 